\title{
The effect of the ultraviolet part of the gluon propagator on the heavy quark propagator
}

\author{
C. J. Burden \\ Department of Theoretical Physics, Research School of Physical Sciences and Engineering, \\ Australian National University, Canberra, ACT 0200, Australia
}

\begin{abstract}
We revisit our recently proposed formalism for dealing with the heavy quark limit of the quark Dyson-Schwinger equation. An ambiguity inherent in the original version of method is identified and resolved. Our reanalysis illustrates the importance of correctly accounting for the effect of hard gluon momenta on the heavy quark self energy in the vicinity of the bare fermion mass pole.
\end{abstract}

PACS NUMBERS: 12.38.Aw, 12.38.Lg, 12.39.Hg 


\section{INTRODUCTION}

In a previous paper [1] we have developed a formalism for dealing with the quark DysonSchwinger equation (DSE) in the heavy quark limit $m_{R} \rightarrow \infty$ where $m_{R}$ is the renormalised heavy quark mass. The purpose of this brief report is to point out an ambiguity in the limiting procedure described in the previous paper, and to illustrate the procedure for extracting the correct heavy quark limit. The ambiguity arises from failing to take proper account of the contribution of hard gluon momenta to the loop integral in the DSE. We begin with a brief summary of the renormalised DSE.

\section{DYSON-SCHWINGER EQUATION}

Our starting point is the renormalised quark DSE [2]

$$
\Sigma^{\prime}(p, \Lambda)=Z_{1}\left(\mu^{2}, \Lambda^{2}\right) \frac{4 g^{2}}{3} \int^{\Lambda} \frac{d^{4} q}{(2 \pi)^{4}} D_{\mu \nu}(p-q) \gamma_{\mu} S(q) \Gamma_{\nu}(q, p)
$$

where we have used a Euclidean metric in which timelike vectors satisfy $p^{2}=-p_{\text {Minkowski }}^{2}<0$, and for which $\left\{\gamma_{\mu}, \gamma_{\nu}\right\}=2 \delta_{\mu \nu}$. We write the renormalised quark propagator in the form

$$
S(p, \mu)=\frac{1}{i \gamma \cdot p A\left(p^{2}, \mu^{2}\right)+B\left(p^{2}, \mu^{2}\right)}=-i \gamma \cdot p \sigma_{V}\left(p^{2}, \mu^{2}\right)+\sigma_{S}\left(p^{2}, \mu^{2}\right),
$$

where $\sigma_{V}\left(p^{2}\right)=A\left(p^{2}\right) /\left(p^{2} A^{2}+B^{2}\right)$ and $\sigma_{S}\left(p^{2}\right)=B\left(p^{2}\right) /\left(p^{2} A^{2}+B^{2}\right)$. The unrenormalised self energy is written

$$
\Sigma^{\prime}(p, \Lambda)=i \gamma \cdot p\left[A^{\prime}\left(p^{2}, \Lambda^{2}\right)-1\right]+B^{\prime}\left(p^{2}, \Lambda^{2}\right)
$$

where renormalised and bare quantities are related by

$$
\begin{gathered}
A\left(p^{2}, \mu^{2}\right)=1+A^{\prime}\left(p^{2}, \Lambda^{2}\right)-A^{\prime}\left(\mu^{2}, \Lambda^{2}\right), \\
B\left(p^{2}, \mu^{2}\right)=m_{R}\left(\mu^{2}\right)+B^{\prime}\left(p^{2}, \Lambda^{2}\right)-B^{\prime}\left(\mu^{2}, \Lambda^{2}\right) .
\end{gathered}
$$

The renormalisation scale is set such that

$$
\left.S(p)^{-1}\right|_{p^{2}=\mu^{2}}=i \gamma \cdot p+m_{R}\left(\mu^{2}\right)
$$

The set of equations (2.1) to (2.6) together with the 'abelian approximation' $Z_{1}=Z_{2}$ can be solved numerically for the propagator functions $A$ and $B$ once the renormalised quark-gluon vertex function $\Gamma_{\mu}$, renormalised gluon propagator $D_{\mu \nu}$, renormalisation point $\left(\mu, m_{R}(\mu)\right)$ and cutoff $\Lambda$ are specified.

In a general covariant gauge, the gluon propagator takes the form

$$
g^{2} D_{\mu \nu}(k)=\left(\delta_{\mu \nu}-\frac{k_{\mu} k_{\nu}}{k^{2}}\right) \Delta\left(k^{2}\right)+g^{2} \xi \frac{k_{\mu} k_{\nu}}{k^{4}},
$$


where $\xi$ is the gauge fixing parameter. In this note we shall assume the bare vertex or "rainbow" approximation for the vertex function, namely $\Gamma_{\mu}(p, q)=\gamma_{\mu}$.

Using Dirac trace identities to project out from Eqs. (2.1) to (2.3) a pair of coupled integral equations gives

$$
\begin{aligned}
A^{\prime}\left(p^{2}, \Lambda^{2}\right)=1 & +\frac{4 Z_{1}}{3 p^{2}} \int^{\Lambda} \frac{d^{4} q}{(2 \pi)^{4}}\left\{\left[p \cdot q+2 \frac{p \cdot(p-q) q \cdot(p-q)}{(p-q)^{2}}\right] \Delta\left[(p-q)^{2}\right]\right. \\
+ & \left.\xi\left[p \cdot q-2 \frac{p \cdot(p-q) q \cdot(p-q)}{(p-q)^{2}}\right] \frac{g^{2}}{(p-q)^{2}}\right\} \sigma_{V}\left(q^{2}\right), \\
B^{\prime}\left(p^{2}, \Lambda^{2}\right) & =\frac{4}{3} Z_{1} \int^{\Lambda} \frac{d^{4} q}{(2 \pi)^{4}}\left\{3 \Delta\left[(p-q)^{2}\right]+\xi \frac{g^{2}}{(p-q)^{2}}\right\} \sigma_{S}\left(q^{2}\right) .
\end{aligned}
$$

\section{THE HEAVY QUARK LIMIT}

The heavy quark limit is taken by assuming the expansions

$$
A\left(p^{2}, \mu^{2}\right)=1+\frac{\Sigma_{A}(K, \kappa)}{m_{R}\left(\mu^{2}\right)}, \quad B\left(p^{2}, \mu^{2}\right)=m_{R}\left(\mu^{2}\right)+\Sigma_{B}(K, \kappa),
$$

where we have defined the new momentum variable $K$ and renormalisaton point $\kappa$ by

$$
K=\frac{p^{2}+m_{R}^{2}}{2 i m_{R}}, \quad \kappa=\frac{\mu^{2}+m_{R}^{2}}{2 i m_{R}} .
$$

The change of independent variable $p^{2} \rightarrow K$ is illustrated in Fig. 1 of Ref. [1]. Our aim is to solve for the quark propagator in the vicinity of $p^{2}=-m_{R}^{2}$, or equivalently, $K=0$. From Eq. 3.1 we have

$$
m_{R} \sigma_{V}\left(p^{2}\right)=\frac{1}{2} \sigma_{Q}(K)+O\left(\frac{1}{m_{R}}\right), \quad \sigma_{S}\left(p^{2}\right)=\frac{1}{2} \sigma_{Q}(K)+O\left(\frac{1}{m_{R}}\right)
$$

where we have defined

$$
\sigma_{Q}(K)=\frac{1}{i K+\Sigma(K)}, \quad \text { and } \quad \Sigma(K)=\Sigma_{B}(K)-\Sigma_{A}(K) .
$$

Note that Eq. (3.3) is valid in any part of the complex momentum plane in which Eq. (3.1) holds, that is, there is no assumption that we are in the vicinity of $K=0$.

For the purposes of expanding the DSE in the inverse quark mass we introduce the momentum substitutions generic to heavy quark effective theory

$$
p_{\mu}=i m v_{\mu}+k_{\mu}, \quad q_{\mu}=i m v_{\mu}+k_{\mu}^{\prime},
$$

where $v_{\mu} v_{\mu}=1$. Typically we take $v_{\mu}=(0,0,0,1)$, which entails $K=k_{4}+O\left(1 / m_{R}\right)$ in the vicinity of $p^{2}=-m_{R}^{2}$. Note also that the result $S(p, \mu)=\frac{1}{2}\left(1+\gamma_{4}\right) \sigma_{Q}(K, \kappa)+O(1 / m)$ for the dressed quark propagator is only a good approximation for $\left|k_{\mu}\right|<<m_{R}$. 
In Ref. [1] the heavy quark DSE is obtained by substituting Eqs. 3.3) and (3.5) into Eqs.(2.8) and (2.9), and then using Eqs. (2.4) and (2.5) to obtain an integral equation for the heavy quark self energy $\Sigma(K)$. The procedure involves truncating a $1 / m_{R}$ expansion of the integrand of the DSE. However, for heavy quarks, and for a model gluon propagator with a realistic ultraviolet tail, the DSE receives contributions from values of $k_{\mu}^{\prime}$ (defined in Eq. (3.5)) which are not small compared with $m_{R}$. Because of this the $1 / m_{R}$ expansion must be applied with some caution.

To understand in detail what can go wrong with the procedure, consider the model gluon propagator used in ref. [1], namely the smeared Frank and Roberts propagator

$$
\Delta\left(k^{2}\right)=(2 \pi)^{4} \frac{m_{t}^{2} d}{\alpha^{2} \pi^{2}} e^{-k^{2} / \alpha}+4 \pi^{2} d \frac{1-e^{-k^{2} /\left(4 m_{t}^{2}\right)}}{k^{2}}=\Delta_{\mathrm{IR}}\left(k^{2}\right)+\frac{4 \pi^{2} d}{k^{2}},
$$

where $d=12 /\left(33-2 N_{f}\right), N_{f}=3$ is the number of light quark flavours, $m_{t}=0.69 \mathrm{GeV}$ is a parameter fitted to a range of calculated pion observables, and the Gaussian width $\alpha$ was chosen to be $0.5643(\mathrm{GeV})^{2}$. As we have indicated, this propagator has the appearance of a Coulomb propagator beyond the scale of the parameter $m_{t}$. Eq. (2.8) then becomes

$$
\begin{aligned}
A^{\prime}\left(p^{2}, \Lambda^{2}\right)= & 1+\frac{4 Z_{1}}{3 p^{2}} \int^{\Lambda} \frac{d^{4} q}{(2 \pi)^{4}}\left\{\left[p \cdot q+2 \frac{p \cdot(p-q) q \cdot(p-q)}{(p-q)^{2}}\right] \Delta_{\mathrm{IR}}\left[(p-q)^{2}\right] \sigma_{V}\left(q^{2}\right)\right. \\
& \left.+4 \pi^{2} d\left[\left(1+\xi^{\prime}\right) p \cdot q+2\left(1-\xi^{\prime}\right) \frac{p \cdot(p-q) q \cdot(p-q)}{(p-q)^{2}}\right] \frac{\sigma_{V}\left(q^{2}\right)}{(p-q)^{2}}\right\}
\end{aligned}
$$

where $\xi^{\prime}=g^{2} \xi /\left(4 \pi^{2} d\right)$. The procedure employed in Ref. [1] of taking the heavy quark limit by substituting Eq. (3.5) and keeping only leading order in $1 / m_{R}$ contributions to the integrand is certainly valid for the term containing $\Delta_{\mathrm{IR}}$. This is because the exponentially damped gluon propagator ensures that the factor in square brackets is only sampled for $k_{\mu}^{\prime}<<m_{R}$. For the remaining Coulomb part, however, one runs into problems.

Consider for a moment the identity

$$
0=\int \frac{d^{4} q}{(2 \pi)^{4}}\left[p \cdot q+2 \frac{p \cdot(p-q) q \cdot(p-q)}{(p-q)^{2}}\right] \frac{\sigma_{V}\left(q^{2}\right)}{(p-q)^{2}}
$$

One easily checks that the integrand of Eq. (3.8) is equal to

$$
I(|p|,|q|, \theta)=\frac{|p||q| \sigma_{V}\left(q^{2}\right)}{\sin ^{2} \theta} \frac{d}{d \theta}\left[\frac{\sin ^{3} \theta}{(p-q)^{2}}\right],
$$

where $|p|=(p \cdot p)^{1 / 2},|q|=(q \cdot q)^{1 / 2}$ and $\theta$ is the angle between $p_{\mu}$ and $q_{\mu}$ in Euclidean 4space. Clearly the angular part of the integral, namely $\int I(|p|,|q|, \theta) \sin ^{2} \theta d \theta$, is identically zero. In Landau gauge, $\xi^{\prime}=0$, the second term in Eq. (3.7) should therefore integrate to zero because of cancellations of significant contributions from all directions in Euclidean momentum space. In terms of the Feynman diagram corresponding to the original Euclidean DSE, Eq. (2.1), this means that in the heavy quark limit hard momentum from the incoming quark line can flow through the gluon propagator line, significantly altering the direction of the internal quark line. Considerable cancellations occur in the integration over angles, resulting in a diminished net contribution to Eq. (3.7). 
Inserting the change of momentum variables Eq. (3.5) into the last term in Eq. (3.7) we shall see below that these cancellations do not necessarily occur order by order in $1 / m_{R}$. In order to construct a viable formalism for extracting the heavy quark limit, it is therefore necessary to subtract off the angular oscillatory behaviour of the integrand before expanding in the inverse quark mass.

To this end, we add to Eq. (3.7) an arbitrary multiple of the identity Eq. (3.8) to give

$$
A^{\prime}\left(p^{2}, \Lambda^{2}\right)=1+(\text { infrared part })+\frac{16 \pi^{2} Z_{1} d}{3 p^{2}} \int^{\Lambda} \frac{d^{4} q}{(2 \pi)^{4}} H(p, q) \frac{\sigma_{V}\left(q^{2}\right)}{(p-q)^{4}}
$$

where

$$
H(p, q)=\left(1+\xi^{\prime}+\lambda\right) p \cdot q(p-q)^{2}+2\left(1-\xi^{\prime}+\lambda\right) p \cdot(p-q) q \cdot(p-q),
$$

with $\lambda$ arbitrary.

We can now make use of Eqs. (3.3) and (3.5) to extract from Eqs. (2.4), (2.5), (2.9) and (3.10) an integral equation for $\Sigma(K, \kappa)$. For example, in Landau gauge $(\xi=0)$, and choosing $k_{\mu}=(0,0,0, K)$ (which is allowable to zeroth order in $1 / m_{R}$ provided $K<<m_{R}$ ), we obtain

$$
\begin{aligned}
\Sigma(K, \kappa)= & \frac{4}{3} \int^{\Lambda} \frac{d^{4} k^{\prime}}{(2 \pi)^{4}} \frac{1}{i k_{4}^{\prime}+k^{\prime 2} /\left(2 m_{R}\right)+\Sigma\left(k_{4}^{\prime}, \kappa\right)} \\
& \times\left\{\frac{\left|\mathbf{k}^{\prime}\right|^{2} \Delta_{\mathrm{IR}}\left[\left(K-k_{4}^{\prime}\right)^{2}+\left|\mathbf{k}^{\prime}\right|^{2}\right]}{\left(K-k_{4}^{\prime}\right)^{2}+\left|\mathbf{k}^{\prime}\right|^{2}}-\right. \\
& {\left.\left.\left[\frac{3 \lambda}{2}-(1+\lambda) \frac{\left|\mathbf{k}^{\prime}\right|^{2}}{\left(K-k_{4}^{\prime}\right)^{2}+\left|\mathbf{k}^{\prime}\right|^{2}}\right] \frac{4 \pi^{2} d}{\left(K-k_{4}^{\prime}\right)^{2}+\left|\mathbf{k}^{\prime}\right|^{2}}\right\}-(K \rightarrow \kappa)\right) . }
\end{aligned}
$$

The $\lambda=0$ case was solved numerically in Ref. [1]. For arbitrary $\lambda$, however, we find that the solutions are heavily dependent on the irrelevant parameter $\lambda$, so something is clearly amiss.

As pointed out above, and in the Appendix to Ref. [1], the integrand of this equation is sampled for values of $k^{\prime}$ which are not necessarily small compared with $m_{R}$. The terms $\sigma_{S}\left(q^{2}\right) /(p-q)^{2}$ and $\sigma_{V}\left(q^{2}\right) /(p-q)^{4}$ in the Coulomb parts of integrands of Eqs. (2.9) and (3.10) cannot be the cause of the problem, since, as pointed out below Eq. (3.3), they are well represented by the heavy quark approximations $\frac{1}{2} \sigma_{Q} /\left(k-k^{\prime}\right)^{2}$ and $\frac{1}{2} \sigma_{Q} /\left[m_{R}\left(k-k^{\prime}\right)^{4}\right]$ throughout the complex momentum plane. The problem must therefore lie solely with truncation of the $1 / m_{R}$ expansion of $H(p, q)$ in the integrand of Eq. (3.10). By careful choice of the parameter $\lambda$ however, we find that we can subtract of the troublesome angular oscillatory behaviour hidden in the identity (3.8) to leave an integrand which can be accurately expanded in the inverse quark mass even for large values of $k^{\prime}$.

In the Appendix we show that, with $k_{\mu}=(0,0,0, K)$, the parameter choice

$$
\lambda=\xi / 3-1
$$

implies

$$
H(p, q)=-\frac{4 \xi^{\prime} m_{R}^{2}}{3}\left|\mathbf{k}^{\prime}\right|^{2}\left[1+O\left(\frac{K}{m_{R}}\right)\right]
$$


We are interested in solving the DSE equation in the vicinity of the bare quark mass pole, that is, for $|K|<<m_{R}$. In this case, as $m_{R} \rightarrow \infty$, the non-leading terms in the $1 / m_{R}$ expansion of $H$ do not contribute to the integral in Eq. (3.10) and our procedure for obtaining the heavy quark limit is valid. For any other value of $\lambda$ however, the $1 / m_{R}$ expansion of $H$ picks up $O\left(k^{\prime} / m_{R}\right)$ pieces which contribute to the ultraviolet part of the integral and cannot be neglected. This is the source of the discrepancy.

In Fig. [ is plotted the modulus of the heavy quark propagator obtained by solving Eq. (3.12) in Landau gauge with parameter value $\lambda=-1$, consistent with Eq. (3.13). For comparison, we also plot the solution from Ref. [1] corresponding to $\lambda=0$. With the correct value of $\lambda$, conjugate poles have moved considerably deeper into the complex momentum plane, and away from the imaginary $K$ axis, or equivalently, away from the real, timelike $p^{2}$ axis. Very little evidence is left of the original bare propagator mass pole at $K=0$.

\section{COMMENT ON EUCLIDEAN AND MINKOWSKI METRICS}

Our formalism presupposes the quark DSE to be formulated in Euclidean space. This is standard procedure in most nonperturbative treatments of confining field theory including DSE calculations [2]. Opinions differ regarding the question of whether this procedure is simply a matter of practical expediency, or whether it reflects a deeper truth about the nature of confining theories. In its most extreme form 佃, the position adopted is that the underlying quark degrees of freedom can only be meaningfully considered in Euclidean space. If we adopt a less extreme point of view, it may be instructive to explore whether the angular oscillatory function in the integrand of Eq. (3.8) finds its analogue in a Minkowski formalism of the quark DSE. That is, does the quark self energy contain significant contributions from different directions in physical Minkowski space time which cancel?

Consider the case where the Minkowski space vectors $p^{\mu}$ and $q^{\mu}$ are both timelike. We define $|p|=\left(p^{\mu} p_{\mu}\right)^{1 / 2}$ and $|q|=\left(q^{\mu} q_{\mu}\right)^{1 / 2}$. Choosing $p^{\mu}=(|p|, \mathbf{0})$ and $q^{\mu}=$ $(\eta|q| \cosh \theta, \hat{\mathbf{q}}|q| \sinh \theta)$ where $\eta= \pm 1$ and $\theta$ is real, the integrand of Eq. (3.8) becomes

$$
\left[p \cdot q+2 \frac{p \cdot(p-q) q \cdot(p-q)}{(p-q)^{2}}\right] \frac{\sigma_{V}\left(q^{2}\right)}{(p-q)^{2}}=\frac{\eta|p||q| \sigma_{V}\left(q^{2}\right)}{\sinh ^{2} \theta} \frac{d}{d \theta}\left(\frac{\sinh ^{3} \theta}{|p|^{2}+|q|^{2}-2 \eta|p||q| \cosh \theta}\right),
$$

and the integration measure within the forward and backward light cones becomes

$$
\int d^{4} q=\sum_{\eta= \pm 1} \int_{0}^{\infty}|q|^{3} d|q| \int_{0}^{\infty} \sinh ^{2} \theta d \theta \int d \Omega .
$$

The analogue of the integrand over angles at fixed $|q|$, which is zero in the Euclidean formalism, is now

$$
\sum_{\eta= \pm 1} \int_{0}^{\infty} d \theta \frac{d}{d \theta}\left(\frac{\eta \sinh ^{3} \theta}{|p|^{2}+|q|^{2}-2 \eta|p||q| \cosh \theta}\right)
$$

It is difficult to conceive of a regularisation procedure which could render such a severely divergent integral equal to zero. A similar analysis of the case where $p_{\mu}$ is timelike and $q_{\mu}$ 
is spacelike also leads to a strongly divergent integral. We are led to the conclusion that the subtraction procedure described in the previous section is only relevant to the Euclidean formalism.

\section{CONCLUSIONS}

We have carried out a reanalysis of the quark DSE in the heavy mass limit $m_{R} \rightarrow \infty$. It is observed that, for a model gluon propagator with a realistic asymptotic ultraviolet tail, the procedure used in our previous analysis [1] may not lead to a unique solution for the dressed heavy quark propagator in the vicinity of the bare fermion mass pole. The problem lies with failing to account adequately for the contribution to the DSE loop integral from the ultraviolet part of the gluon propagator. In essence, this means that in the Euclidean formalism, the momentum of an intermediate quark state cannot be assumed to be aligned closely with that of the external quark line in the heavy quark limit.

We have solved this problem by adding to the integrand of the DSE a function which integrates to zero over angles in Euclidean space, but which cancels off higher order in $1 / m_{R}$ contributions to the integrand that would otherwise contribute to the zeroth order quark self energy. We note that the analogous function in Minkowski space, when integrated over the two sheeted hyperboloid defined by a constant value for $q^{\mu} q_{\mu}$, is a strongly divergent integral, suggesting that our procedure is unique to the Euclidean formalism. In fact, if such a procedure proved to be necessary and valid in Minkowski space, it would bring into question the notion that backward propagating intermediate states can be ignored in the heavy quark limit. In the absence of a viable nonperturbative treatment of quantum chromodynamics directly formulated in Minkowski space, it is difficult to place a physical interpretation on a procedure which appears to be simply a necessary mathematical step within the Euclidean formalism.

The practical effect of the correction is to shift conjugate propagator poles present in our previous solution deeper into the complex momentum plane, and further from the real, timelike axis. Our solution for the heavy quark propagator can be regarded as the first step in determining the Bethe-Salpeter (BS) amplitude for $Q-\bar{q}$ mesons. In reference [3] it was found that, if a simple Gaussian gluon propagator which does not accurately model the ultraviolet gluon behaviour is used, the pole in the heavy quark propagator is only slightly shifted from its bare propagator position at $K=0$. It was further shown that the presence of this pole prevented solutions to the ladder approximation $Q-\bar{q}$ BS equation. The removal of propagator poles to remote parts of the complex plane exhibited by our corrected solutions should overcome this difficulty.

A calculation of the Isgur-Wise function using an impulse approximation involving DSE inspired light quark propagators and bare heavy quark propagators has been carried out in reference [5]. In this calculation the bound state $Q-\bar{q}$ BS amplitude was modelled using a variety of Gaussian-like functional forms, each using a single fitted scale parameter. It was found that the calculated Isgur-Wise function is insensitive to which functional form used provided a suitable choice was made for the scale parameter. The appropriateness of the fitting procedure employed in reference [5] can be tested once the next step of modelling the BS equation with the corrected heavy quark propagator is completed. 


\section{ACKNOWLEDGEMENT}

The author acknowledges helpful discussions with F. T. Hawes and C. D. Roberts, and the hospitality of the Special Research Centre for the Subatomic Structure of Matter in Adelaide.

\section{APPENDIX: EXPANSION OF $H$}

Inserting Eq. (3.5) into Eq. (3.11) we obtain

$$
H(p, q)=H_{2}(p, q) m_{R}^{2}+H_{1}(p, q) m_{R}+H_{0}(p, q),
$$

where, after setting $k_{\mu}=(0,0,0, K)$,

$$
\begin{aligned}
H_{2}= & (1+\lambda)\left[-3\left(k_{4}^{\prime}-K\right)^{2}-\left|\mathbf{k}^{\prime}\right|^{2}\right]+\xi^{\prime}\left[\left(k_{4}^{\prime}-K\right)^{2}-\left|\mathbf{k}^{\prime}\right|^{2}\right], \\
H_{1}= & i(1+\lambda)\left[3 K^{3}-3 K^{2} k_{4}^{\prime}-K k^{\prime 2}+3 k^{\prime 2} k_{4}^{\prime}-2 K k_{4}^{\prime 2}\right] \\
& +i \xi^{\prime}\left[-K^{3}+K^{2} k_{4}^{\prime}+3 K k^{\prime 2}-k^{\prime 2} k_{4}^{\prime}-2 K{k_{4}^{\prime}}^{2}\right], \\
H_{0}= & (1+\lambda)\left[3 K^{3} k_{4}^{\prime}+3 K k^{\prime 2} k_{4}^{\prime}-2 K^{2} k^{\prime 2}-4 K^{2}{k_{4}^{\prime}}^{2}\right]+\xi^{\prime}\left[-K^{3} k_{4}^{\prime}-K k^{\prime 2} k_{4}^{\prime}+2 K^{2} k^{\prime 2}\right] .
\end{aligned}
$$

The $k^{\prime 2} k_{4}^{\prime}$ term in $H_{1}$ and the $K k^{\prime 2} k_{4}^{\prime}$ in $H_{0}$ can be disposed of by choosing $\lambda=\xi / 3-1$, which reduces $H(p, q)$ to

$$
H(p, q)=-\frac{4 \xi^{\prime} m_{R}^{2}}{3}\left|\mathbf{k}^{\prime}\right|^{2}\left(1-\frac{2 i K}{m_{R}}-\frac{K^{2}}{m_{R}^{2}}\right) .
$$

For the region $|K|<<m_{R}$, the final two terms can be ignored without affecting the solution of the DSE to zeroth order in $1 / m_{R}$. 


\section{REFERENCES}

[1] C. J. Burden, Phys. Rev. D 57, 276 (1998).

[2] C. D. Roberts and A. G. Williams, Prog. Part. and Nucl. Phys. 33, 475 (1994).

[3] C. J. Burden and D.-S. Liu, Phys. Rev. D 55, 367 (1997).

[4] S. J. Stainsby, and. R. T. Cahill, Phys. Lett. A146 467 (1990).

[5] M. A. Ivanov, et al., Phys. Lett. B 416, 29 (1998); Phys. Rev. C 57, 1991 (1998). 


\section{FIGURES}

FIG. 1. The modulus $\left|\sigma_{Q}(K)\right|$ of the heavy quark obtained by solving the heavy quark DSE Eq. (3.12) in rainbow approximation with a Landau gauge, gaussian smeared Frank and Roberts gluon propagator. The renormalised heavy quark mass is $m_{R}=5 \mathrm{GeV}$, and remaining input parameter values are given in the text. The steeper surface with an obvious pole is the solution

found in Ref. [1] corresponding to $\lambda=0$. The shallower surface is the solution corresponding to the correct parameter value, $\lambda=-1$. 


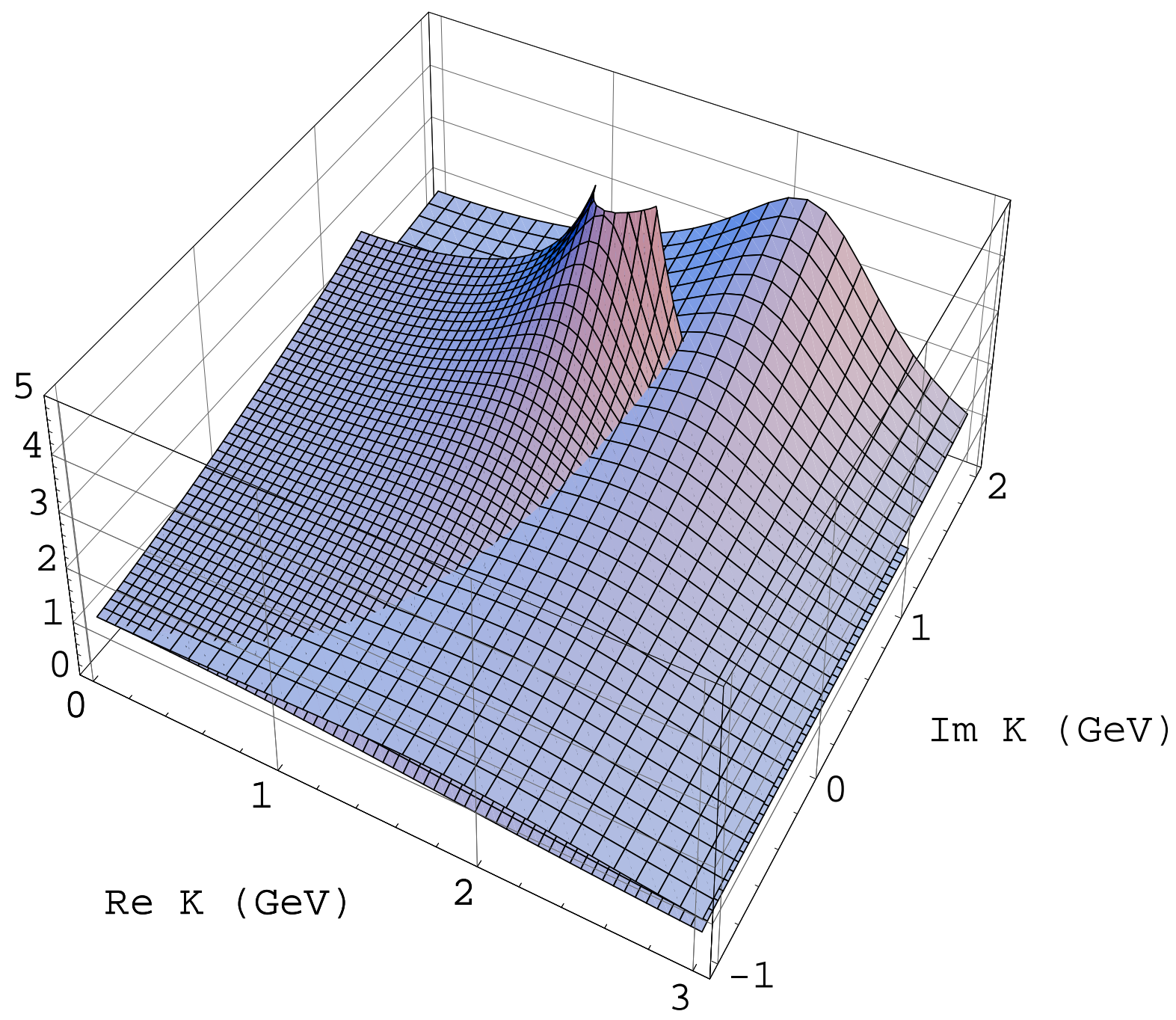

